\title{
Modelling the effect of hydration on skin conductivity
}

\author{
Luke Davies $^{1,2}$, Paul Chappell ${ }^{2}$ and Tracy Melvin ${ }^{1,3}$ \\ ${ }^{1}$ Institute of Complex Systems and Simulation, University of Southampton, Southampton, SO17 \\ 1BJ, UK \\ ${ }^{2}$ Electronics and Computer Science, University of Southampton, Southampton, SO17 1BJ, UK \\ ${ }^{3}$ Optoelectronics Research Centre, University of Southampton, Southampton, SO17 1BJ, UK
}

\begin{abstract}
A model representing the skin was subjected to a variety of electrical signals. The parts of the model representing the Stratum Corneum were given different conductivities to represent different levels of hydration. The overall impedance and conductivity of the cells did not vary at frequencies below $40 \mathrm{kHz}$. Above $40 \mathrm{kHz}$, levels of conductivity caused the overall impedance to decrease. The variation in impedance with conductivity between $5 \mathrm{mSm}^{-1}$ and $50 \mathrm{mSm}^{-1}$ can be modelled quadratically while variation in impedance with conductivity between $5 \mathrm{mSm}^{-1}$ and 5000 $\mathrm{mSm}^{-1}$ can be modelled with a double exponential decay.
\end{abstract}

\section{Keywords}

Skin conductance - Skin potential - Modelling - Bioimpedance

\section{Introduction}

There are a number of different applications for which being able to accurately determine the behaviour of the body from an electrical point of view is useful. These applications are relevant to areas such as biochemistry and biophysics and they are highly relevant for a number of techniques used in medicine. Being able to send and receive signals from the body without having to do an invasive procedure, using the circuits shown in figure 1 , opens up a number of relatively safe techniques that can be used for diagnosis as well as rehabilitate a variety of conditions $[1,2]$.

Of particular interest is using knowledge of the electrical impedance of skin between two electrodes to improve the efficacy of functional electrical stimulation (FES) [3-5]. FES is the process of passing electrical current into the body through the skin in order to generate contractions in paralysed muscles; a typical waveform is shown in figure 2. To voluntarily move a muscle, signals are sent from the brain through the nervous system to the desired motor neurons to cause contractions in the muscle fibres. The motor neurons and their innervated muscle fibres form a motor unit. Muscles that create large movements such as the leg muscles or arm muscles have few motor units each containing a large number of muscle fibres, while the muscles involved in fine movement, such as facial movement, have motor units that only contain a few muscle fibres.

Figure 1. Circuits used for sending electrical current into skin (left) or recording signals from the body (right)

By using FES it is possible to externally activate motor units that cannot be activated voluntarily. A common example of where FES is employed is for people who have suffered a stroke resulting in foot drop. Foot drop is 
when the foot does not lift properly when walking during the swing phase, causing the toes to drag on the ground. The foot is unable to lift due to either weakness or paralysis in the muscles that lift the foot. FES can be used to try and stimulate externally afferent and efferent nerves associated with the affected muscles causing the foot to lift.

One of the difficulties faced with FES is being able to activate only the desired motor units and in such a way as to make the muscle movements feel as natural as possible. To this end, knowing how the skin will affect a stimulation pulse is important to be able to activate the desired motor units.

Figure 2. An illustration of a waveform used in FES. A usual waveform will have a frequency of $20-40 \mathrm{~Hz}$, amplitude of $0-120$ $\mathrm{mA}$, applied voltage up to $120 \mathrm{~V}$ and pulse duration of 0-300 $\mu \mathrm{S}$. A biphasic waveform is used to both introduce and remove charge [6].

\subsection{Skin Structure}

In order to determine the dielectric properties of skin, the structure of skin should be considered. There are two main layers that make up skin, the epidermis and the dermis, as well as a layer between the skin and the underlying bone and muscle called the hypodermis. The thickness of skin varies depending on the location on the body being considered. The skin is thickest on the palms and soles of the feet at around $4 \mathrm{~mm}$ thick and thinnest around the eyelids at 0.5 $\mathrm{mm}$. These values are not exact and will vary from person to person [7-9].

The epidermis is the outermost layer of skin and serves as a chemical and diffusional barrier. The epidermis regulates the amount of water released from the body through transepidermal water loss. It is mainly composed of keratinocytes, accounting for $95 \%$ of the cells present [10-12]. Other types of cell in the epidermis include melanocytes which are responsible for melanin production, Langerhans cells, which are dendritic cells involved in capture, uptake and processing of antigens, and Merkel Cells which are associated with the sense of light touch [13].

The epidermis itself is composed of a number of separate layers, as shown in figure 3. Each of these layers serves a different purpose. The most exposed layer is stratum corneum (SC) which acts as a diffusion barrier [14]. The next layer down is the stratum lucidum that exists only in locations that are exposed to substantial friction, such as the palms and soles. Below the stratum lucidum is the stratum granulosum that provides the SC with dead cells. In the stratum spinosum the Langerhans cells are located. The final layer is the stratum basale, which is a continuous layer of basal keratinocytes that is normally only one cell thick [13].

Figure 3. Structure of the epidermis

From an electrical point of view one of the most important sub layers is the stratum corneum. The stratum corneum is the top layer of the epidermis and the top layer of skin as a whole. The stratum corneum is a thin layer made up of dead skin cells being only around 20 microns thick on average; it is the most insulating layer due to the lack of moisture in the cells. This high level of insulation causes the stratum corneum to play a dominant role in the skin's electrical response when low frequency electric fields and DC current are applied. However, the 
dominant effect of the SC is heavily influenced by hydration as well as other factors such as the electrode geometry and size $[10,16-18]$.

\subsection{Skin Cells}

The stratum corneum comprises very thin hexagonal or pentagonal cells, the corneocytes. They contain very dense keratin. They overlap like a brick wall and are held together with the corneodesmosomes acting as rivets to maintain the rigid structure. Between the cells there is a complex array of lipids, ceramides, free fatty acids, cholesterol and cholesterol sulphate.

While the cell membrane plays the significant role in the behaviour of the cell, the inner parts of the cell also has an impact in the electrical properties of a cell. There has in fact been a number studies into how cells interact with one another and what role electrical signals play in these interactions. However, it is generally thought that these interactions have only a small effect on the cells overall electrical behaviour and so for the purposes of the model the cells inner parts are treated as having uniform dielectric properties with no connection between cells [19-21].

\subsection{Electrical properties of skin}

Experimentally, two types of circuit were used originally in order to acquire the electrical properties of skin. The simplest circuit involved two electrodes that would provide information about the $D C$ response of skin. A more complicated four electrode circuit was used to determine the $D C$ and $A C$ response of skin. Both circuits relied upon comparing the skin to a reference voltage from which the skin's impedance can be obtained. The most common way for the impedance to be measured in recent times is to simply use an impedance analyser, which can provide the required values for the dielectric properties of skin without the need for creating specialised circuitry [22-25].

\subsection{Hydration}

One of the most significant factors that can alter the skin's impedance is hydration; in fact measuring the electrical impedance of skin can be used in order to determine the water content of the SC in vivo. A standard measure for hydration is water activity $\left(a_{w}\right)$ :

$$
a_{w}=p / p_{0}
$$

where water activity $\left(a_{w}\right)$ is the partial vapour pressure of water in a substance $(p)$ divided by the standard state partial vapour pressure of water $\left(p_{0}\right)$ [26]. A paper by Börklund et al [26] found that when exposed to a hydrating solution, $a_{w}=0.992$, the resistance of the skin is on average 14 times lower with the effective capacitance is 1.5 times higher when compared with dehydrating conditions, $a_{w}=0.826$. These changes are largely reversible and have been tested over a 96 hour period.

Knowing the effect of hydration is also important because tap-water is sometimes used in practice to improve the electrical connection between electrodes and skin.

\subsection{Electrical properties of Cells}

An alternative approach is to consider the skin as a collection as cells instead of a bulk approach considered so far. By considering the cells themselves it may be possible to produce a high level model that more accurately represents the electrical response of skin and other parts of the body. There 
have been a number of papers produced exploring how single cells react to an electric field. While most of the cells tested are not skin cells there is still plenty of relevant information due to the similarity between cells. One of the main drawbacks of this approach is that trying to analyse the response of individual cells that are surrounded by large groups of cells is difficult so there has not been much research in the area. There are about 60 million cells in one square $\mathrm{cm}$ of skin and trying to make a model that included every individual cell would require a significant amount of computational power. While it may be possible to do this, it is difficult to validate such a model since there is not much information about how the interactions of cells affect their electrical response when grouped. While a completely faithful representation might be of questionable value, a smaller model that considers how a small number of cells interact may provide a lot of useful information about the skin's electrical behaviour $[27,28]$.

In order to produce a model of a cell it is important that there is analytical or experimental data to compare a model so that the results can be in some way verified. The first values for a cell's electric properties were obtained by applying an electric field to cells suspended in a conducting medium and measuring the resulting impedance $[29,30]$. This approach allowed for the isolation and testing of single cells but the environment is not a true representation of the medium a cell is usually located in. Using cells in suspension also tend to lead to more regularly shaped cells than those that are present in the body.
Attempting to find a means of acquiring these properties from cells in the body is extremely challenging. There is the small size of the cells, their location and their proximity to other cells. These are also issues involving cells that move, such as blood cells. However, there are techniques available that are able to overcome some of these issues. One such method that can show a change in voltage across a cell membrane is to use special dyes. The intensity of the fluorescence of these dyes changes linearly with the induced transmembrane voltage (ITV). The main downside of such a dye is that they tend to be highly susceptible to noise and the environment, leading to large potential errors in the results [31,32].

\section{Materials and Methods}

A finite element model was produced in order to analyse the effect of hydration of epidermal cells. The model consists of a layer of cells designed to represent the electrical properties of skin cells and a layer of cells that represent the collective behaviour of the other epidermal layers. The number of cells used to represent the depth of the SC was 15 which is how many would be expected from literature[9]. Because of the less significant role played by the lower levels of the epidermis 4 larger cells were used with the same electrical properties.

COMSOL 5.1 software was used to simulate the model. The model was set up as a frequency dependant study using the electric currents module. COMSOL uses finite element methods in order to arrive at an approximate solution for a model given a set of starting parameters and a final desired error tolerance. The designed model is split into small segments known as elements and the program applies the equations in electric 
currents model to simulate the elements as they would in reality. While other modules for mechanics and heat exist it is unlikely the field strengths being used would significantly heat or deform the cells so these modules are not used.

Using the inbuilt CAD tools a 3D model of the surrounding medium was created, the resulting cell can be seen in figure 4 . Each element was then given a relative permittivity and electrical conductivity in keeping with the values for their biological counterparts, with the exact values shown in table 1.

Figure 4. Geometry of the model showing the extracellular medium and the three types of cell used with one layer showing the cornedesomes

In order to reduce the computational power needed to run the simulation the cell membrane was replaced with a boundary condition. The contact impedance is part of the electric currents physics package. This substitution removes the need for a much finer mesh for the nanometre scale membrane compared to the other micrometre scale elements.

Table 1. Parameters for the single cell model

One of the sides of the extracellular medium was set to be the input signal with the opposite side grounded. The remaining sides are set as electrical insulators. The input signal was set up as a sinusoidal voltage that varies between $0 \mathrm{~V}$ and $10 \mathrm{~V}$ and tested at 11 frequencies: $100 \mathrm{~Hz}, 1 \mathrm{kHz}, 10 \mathrm{kHz}, 20 \mathrm{kHz}$, $50 \mathrm{kHz}, 100 \mathrm{kHz}, 200 \mathrm{kHz}, 500 \mathrm{kHz}, 1 \mathrm{MHz}, 5 \mathrm{MHz}$ and $10 \mathrm{MHz}$.
In order to measure the impact of hydrating the skin specifically the SC cytoplasm was simulated with a variety of different conductivities starting from $5 \times 10^{-3} \mathrm{Sm}^{-1}$ increasing in steps of $5 \times 10^{-3} \mathrm{Sm}^{-1}$ up to and including $50 \times 10^{-3} \mathrm{Sm}^{-1}$ to represent the typical conductivity of tap water [33] with one additional conductivity of $1 \times 10^{-7} \mathrm{Sm}^{-1}$ to represent the lowest conductivity that might be expected from a corneocyte. Only the conductivity of the cells in the SC were changed because work done previously has shown that as long as the SC is intact then there is no significant effect of hydration on skin impedance due to tissue fluid below the SC.

\section{Results}

Once the simulations had been run, the voltage gradient across the cells as well as the current flowing through the cells were recorded at each frequency in order to calculate the overall impedance of the model. An example voltage gradient can be seen in Figure 5 and the impedances recorded for the different values of SC cytoplasm conductivity are shown in Figure 7.

The results in figure 6 show that at lower frequencies the conductivity of the cell has no impact on the overall impedance. Above $10^{4} \mathrm{~Hz}$ the the impedences diverge, with the impedance decreasing with a lower conductivity.

Figure 5. Voltage gradient across model with SC conductivity of $0.05 \mathrm{Sm}^{-1}$

In order to explore the effect of hydration at the higher frequencies the simulation was changed to be run at a set of frequencies with a range of different conductivities. The results show that between $50 \mathrm{kHz}$ and $2 \mathrm{MHz}$ there is a change in the impedance of the model that decreases by around $200 \Omega$. When looking specifically at the typical 
conductivities of tap water the results show that for each frequency there is a very slight curve, shown in figure 6 that can be fitted to a quadratic equation

$$
Z=A \sigma^{2}-B \sigma+C
$$

using the parameters shown in table 2 where $Z$ is impedance and $\sigma$ is conductivity fitted using Matlab.

Table 2. Parameters for $A \sigma^{2}-B \sigma+C$

This behaviour was further explored by expanding the range of conductivities tested to include up to $5 \mathrm{Sm}^{-1}$, the typical conductivity of sea water. This shows a very different behaviour with the change in conductivity decreasing significantly slower at above $0.05 \mathrm{Sm}^{-1}$, with almost constant Impedance at $50 \mathrm{kHz}$, shown in figure 7. This change in behaviour can be modelled as an offset double exponential decay,

$$
Z=A e^{B \sigma}+C e^{D \sigma}+E
$$

with parameters shown in table 3 where $Z$ is impedance and $\sigma$ is conductivity fitted using Matlab.

Table 3. Parameters for $A e^{B \sigma}+C e^{D \sigma}+E$

Figure 6. Top plot shows a loglog plot of the Change in impedance of cells with increasing frequency $(\mathrm{Hz})$ with 3 different SC cytoplasm conductivites $\left(\mathrm{Sm}^{-1}\right)$ for signal frequencies between $100 \mathrm{~Hz}$ and $10 \mathrm{MHz}$, bottom plot shows frequencies between $40 \mathrm{kHz}$ and $10 \mathrm{MHz}$ with linear impedance axis

Figure 7. Change Impedance due to varying SC cytoplasm conductivity between $0.005 \mathrm{Sm}^{-}$ ${ }^{1}$ and $0.05 \mathrm{Sm}^{-1}$ at 10 signal frequencies (Top) and 5 signal frequencies (Bottom)

\section{Discussion}

The results of the model show that at high frequencies there is a noticeable change in the impedance of skin. There is a $10-50 \%$ reduction between the typical unhydrated skin impedance and highly conductive water being applied depending on the frequencies used. The results of this model support the idea that hydrating the skin can improve the results obtained clinically by reducing the skin's impedance. Because of the wide range of conductivities that standard tap water can possess it may be that this effect will vary across locations depending on the type of water available. It should also be noted that the hydration of skin will change over time so reapplication of water is necessary for consistent results. Depending on the length of the applied signals and on how frequently the signals are applied there may also be a change in impedance caused by the accumulation of sweat in the sweat glands.

Lowering the conductivity of the SC cytoplasm creates an almost linear drop in impedance. This shows that the effect of SC conductivity in the model diminishes very slightly, which suggests that the higher the conducting material used on the skin the better in order to lower impedance. However this changes once the conductivity is high enough, around $0.02 \mathrm{Sm}^{-1}$ where the impedance decreases very slightly and at lower frequencies does not change. This is likely due to the lower levels becoming the dominant factor in determining the cells overall impedance creating a lower limit for the impedance. Knowing that this lower limit exists has implications for treating people who have been exposed to highly conducting liquids, such as sea water, that might create hazards e.g. in defibrillation. 
There are a number of areas outside of FES that could benefit from knowing how skin impedance affects conductivity. For example, the model could be expanded to look at the effects of applying a much larger voltage to the cells similar to the voltages used in defibrillation. This would also require looking at the effect of high voltages on the electrical properties of skin cells.

It should also be noted that the effect of hydrating skin would not affect all the stratum corneum equally as the outermost cells are the driest and will have a more significant impact on the impedance then the innermost cells in the SC. It is also important to note when the SC has been removed in experimentation then the model does not reflect how the impedance would change since only the hydration of the SC is being taken into account.

Within the range of conductivities from 0.005 $\mathrm{Sm}^{-1}$ to $0.05 \mathrm{Sm}^{-1}$ for a constant frequency above $40 \mathrm{kHz}$ a quadratic model describes the variation of impedance with conductivity. Within the range of conductivities from 0.005 $\mathrm{Sm}^{-1}$ to $0.05 \mathrm{Sm}^{-1}$ for a constant frequency above $40 \mathrm{kHz}$ a double decaying exponential model describes the variation of impedance with conductivity. At frequencies above $3 \mathrm{MHz}$ the exponential model parameters become constant.

The simulation indicates that at high frequencies, above $200 \mathrm{kHz}$, with good hydration the skin impedance can approximately halve, $53 \mathrm{k} \Omega$ to $27 \mathrm{k} \Omega$. 


\section{References}

1. Davie AP, Francis CM, Love MP, Caruana L, Starkey IR, Shaw TRD, et al. Value of the electrocardiogram in identifying heart failure due to left ventricular systolic dysfunction.

Bmj. 1996;312:222.

2. Niedermeyer $E$, da Silva FL.

Electroencephalography: basic principles, clinical applications, and related fields [Internet]. Lippincott Williams \& Wilkins; 2005 [cited 2014 Jul 14]. Available from: http://books.google.co.uk/books?hl=en \&lr=\& $\mathrm{id}=$ tndqYGPHQdEC\&oi=fnd \&pg=PR11\&dq=EI ectroencephalography\&ots=GN5n2505tB\&sig =1qpAzAXKhiHIXM727Obb3oJEsYA

3. Osiri M, Welch V, Brosseau L, Shea B, McGowan JL, Tugwell $P$, et al.

Transcutaneous electrical nerve stimulation for knee osteoarthritis (Review). 2009 [cited 2014 Jun 18]; Available from: http://onlinelibrary.wiley.com/doi/10.1002/1 4651858.CD002823/pdf/standard

4. Butikofer R, Lawrence PD. Electrocutaneous Nerve Stimulation-I: Model and Experiment. IEEE Trans. Biomed. Eng. 1978;BME-25:526-31.

5. Lynch CL, Popovic MR. Functional Electrical Stimulation. IEEE Control Syst. 2008;28:4050.

6. Criswell E. Cram's introduction to surface electromyography [Internet]. Jones \& Bartlett Publishers; 2010 [cited 2014 Jul 14]. Available from:

http://books.google.co.uk/books?hl=en\&lr=\& $\mathrm{id}=\mathrm{ADYm0TqiDo8C} \&$ oi=fnd\&pg $=P P 1 \& \mathrm{dq}=$ Elec tromyography\&ots=RvncNrEs3M\&sig=NC9_2 7MA03InGduxB47xGDSLsHQ

7. Bouwstra JA. The skin barrier, a wellorganized membrane. Colloids Surf. Physicochem. Eng. Asp. 1997;123-124:40313.
8. Coderch L, López O, de la Maza A, Parra JL. Ceramides and skin function. Am. J. Clin.

Dermatol. 2003;4:107-29.

9. Lai-Cheong JE, McGrath JA. Structure and function of skin, hair and nails. Medicine (Baltimore). 2009;37:223-6.

10. Guo YP, Li Y, Yao L, Liu R, Cao XY, Cao ML. Skin Temperature, Stratum corneum water content and transepidermal water loss distributions during running. J Fiber Bioeng Inf. 2011;4:253-66.

11. Harker M. Psychological sweating: a systematic review focused on aetiology and cutaneous response. Skin Pharmacol. Physiol. 2013;26:92-100.

12. ROTH WT, DAWSON ME, FILION DL. Publication recommendations for electrodermal measurements. Psychophysiology. 2012;49:1017-34.

13. Saeed S. Lookingbill \& Marks' Principles of Dermatology [Internet]. LWW; 2007 [cited 2014 Jul 14]. Available from:

http://journals.Iww.com/amjdermatopatholo gy/Abstract/2007/10000/Lookingbill__Mark s_Principles_of_Dermatology.21.aspx

14. Elias PM. The skin barrier as an innate immune element. Semin. Immunopathol. [Internet]. Springer; 2007 [cited 2014 Jul 14]. p. 3-14. Available from:

http://link.springer.com/article/10.1007/s00 281-007-0060-9

15. Kirschner N, Houdek P, Fromm M, Moll I, Brandner JM. Tight junctions form a barrier in human epidermis. Eur. J. Cell Biol.

2010;89:839-42.

16. Menon GK, Cleary GW, Lane ME. The structure and function of the stratum corneum. Int. J. Pharm. 2012;435:3-9.

17. Yamamoto T, Yamamoto Y. Dielectric constant and resistivity of epidermal stratum corneum. Med. Biol. Eng. 1976;14:494-500. 
18. Pliquett U, Langer R, Weaver JC. Changes in the passive electrical properties of human stratum corneum due to electroporation. Biochim. Biophys. Acta BBA - Biomembr. 1995;1239:111-21.

19. Elia S, Lamberti P, Tucci V. A Finite Element Model for The Axon of Nervous Cells. COMSOL Eur. Conf. [Internet]. 2009 [cited 2014 Jun 9]. p. 14-6. Available from: http://www.comsol.fi/papers/6735/downloa d/Elia.pdf

20. Amin M, Dey PP, Badkoobehi H. A complete electrical equivalent circuit model for biological cell. Proc. 7th WSEAS Int. Conf. Appl. Comput. Appl. Comput. Sci. [Internet]. World Scientific and Engineering Academy and Society (WSEAS); 2008 [cited 2014 Jun 18]. p. 343-8. Available from:

http://www.wseas.us/e-

library/conferences/2008/meta_hangzhou/C D/meta-52.pdf

\section{Ahmad MR, Nakajima M, Fukuda T,} Kojima S, Homma M. Single cells electrical characterizations using nanoprobe via ESEMnanomanipulator system. Nanotechnol. 2009 IEEE-NANO 2009 9th IEEE Conf. On [Internet]. IEEE; 2009 [cited 2014 Jun 18]. p. 589-92.

Available from:

http://ieeexplore.ieee.org/xpls/abs_all.jsp?ar number $=5394765$

22. Martinsen OG, Grimnes S. Bioimpedance and Bioelectricity Basics. Academic Press; 2011.

23. Arias LR, Perry CA, Yang L. Real-time electrical impedance detection of cellular activities of oral cancer cells. Biosens. Bioelectron. 2010;25:2225-31.

24. Gabriel C, Gabriel S, Corthout E. The dielectric properties of biological tissues: I. Literature survey. Phys. Med. Biol. 1996;41:2231.
25. Gabriel C, Peyman A, Grant EH. Electrical conductivity of tissue at frequencies below 1 MHz. Phys. Med. Biol. 2009;54:4863.

26. Björklund S, Ruzgas $T$, Nowacka A, Dahi I, Topgaard D, Sparr E, et al. Skin Membrane Electrical Impedance Properties under the Influence of a Varying Water Gradient. Biophys. J. 2013;104:2639-50.

27. Huclova S, Erni D, Fröhlich J. Modelling effective dielectric properties of materials containing diverse types of biological cells. J. Phys. Appl. Phys. 2010;43:365405.

28. Gowrishankar TR, Weaver JC. An approach to electrical modeling of single and multiple cells. Proc. Natl. Acad. Sci.

2003;100:3203-8.

29. Markx GH, Davey CL. The dielectric properties of biological cells at radiofrequencies: applications in biotechnology. Enzyme Microb. Technol. 1999;25:161-71.

30. Gregg EC, Steidley KD. Electrical counting and sizing of mammalian cells in suspension. Biophys. J. 1965;5:393-405.

31. Pucihar G, Miklavcic D, Kotnik T. A timedependent numerical model of transmembrane voltage inducement and electroporation of irregularly shaped cells. Biomed. Eng. IEEE Trans. On. 2009;56:1491501.

32. Pucihar G, Kotnik T, Valič B, Miklavčič D. Numerical determination of transmembrane voltage induced on irregularly shaped cells. Ann. Biomed. Eng. 2006;34:642-52.

33. Light TS, Licht S, Bevilacqua AC, Morash KR. The Fundamental Conductivity and Resistivity of Water. Electrochem. Solid-State Lett. 2005;8:E16. 
Modelling the effect of hydration on skin conductivity

Table 1

\begin{tabular}{cccc}
\hline Object & $\begin{array}{c}\text { Dimensions } \\
(\mu \mathrm{m})\end{array}$ & $\begin{array}{c}\text { Relative } \\
\text { permittivity }\end{array}$ & $\begin{array}{c}\text { Conductivity } \\
\left(\mathrm{Sm}^{-1}\right)\end{array}$ \\
\hline $\begin{array}{c}\text { Extracellular } \\
\text { medium }\end{array}$ & $34 \times 34 \times 19$ & 72 & 1.1 \\
$\begin{array}{c}\text { Cytoplasm } \\
\text { SC Cytoplasm }\end{array}$ & $\begin{array}{c}30 \times 30 \times 30 \\
30 \times 30 \times 1\end{array}$ & 86 & 0.6 \\
$\begin{array}{c}\text { Cell } \\
\text { Membrane }\end{array}$ & 8 & 90 & $1 \times 10^{-7}$ \\
\hline
\end{tabular}


Modelling the effect of hydration on skin conductivity

Table 2

\begin{tabular}{clll}
\hline $\begin{array}{c}\text { Frequency } \\
(\mathrm{MHz})\end{array}$ & $\mathrm{A}$ & $\mathrm{B}$ & $\mathrm{C}$ \\
\hline 1 & 361363 & 66760 & 18440 \\
2 & 324048 & 64502 & 18368 \\
3 & 295464 & 61981 & 18283 \\
5 & 246291 & 56697 & 18067 \\
7 & 204627 & 51358 & 17802 \\
10 & 150657 & 43391 & 17337 \\
\hline
\end{tabular}


Modelling the effect of hydration on skin conductivity

Table 3

\begin{tabular}{cccccc}
\hline $\begin{array}{c}\text { Frequency } \\
(\mathrm{MHz})\end{array}$ & $\mathrm{A}$ & $\mathrm{B}$ & $\mathrm{C}$ & $\mathrm{D}$ & $\mathrm{E}$ \\
\hline 1 & 5862 & -6.26 & 10530 & -22.2 & 38270 \\
2 & 7184 & -3.02 & 13430 & -16.9 & 33230 \\
3 & 8615 & -2.47 & 14340 & -15.3 & 30640 \\
5 & 10390 & -1.95 & 15600 & -13.6 & 27340 \\
\hline
\end{tabular}

